\title{
REFLEXIONS SUR LES TENDANCES ACTUELLES DE LA PSYCHANALYSE DES PSYCHOSES
}

\author{
Darcy Mendonça Uchoa *
}

Le progrès de l'investigation psychanalitique a révélé qu'il n'y a pas de différence fondamentale entre la neurose et la psychose, rendant ainsi possible la compréhension et le traitement des divers types des désordres mentaux au sens restreint. Freud, au début, a fait ressortir le manque de capacité de transfert des psychotiques et de ce fait leur inaccessibilité au point de vue thérapeutique. Une telle opinion ne peut plus être maintenue à l'heure actuelle, puisque, selon l'expérience de la majorité des psychanalystes contemporains, ils ont la même ou peut-être une plus grande capacité de transférer leurs conflicts et leurs problèmes sur le psychanalyste que les neurotiques eux-mêmes. Avec l'analyse du cas Schreber (1911) et l'étude sur le narcissisme (1914), Freud a montré la possibilité et l'utilité de l'application de la méthode d'investigation psychanalitique dans le champ des psychoses.

La modification de la théorie de la libido par K. Abraham et ses études sur la psychose maniaque-dépressive (les états mélancoliques spécialement) ont réprésenté un grand avancement, au début avec ses travaux de 1911 et 1916 (Anzätze zur psychoanalytischen Erforschung und Behandlung des manisch-depressiven Irreseins und verwandter Zustände et Lintersuchungen über die früheste prägenitale Entwicklungstufe der Libido). Ils ont pu être complétés (après l'étude de Freud sur "Trauer und Melancholie") avec le travail définitif intitulé "Versuch einer Entwicklungsgeschichte der Libido auf Grund der Psychoanalyse seelischer Störungen" (1924). Démontrant que le paranoiaque se défend des fantaisies d'inversion sexuelle en utilisant le mécanisme psychologique de projection et postulant la conception que les psychoses sont des maladies narcissiques dans lesquelles "la libido d'objet se transforme en libido du sujet", par des profondes régressions à la phase du narcissisme primaire et avec les phénomènes de restitution le moi cherchant à retrouver les objets du monde extérieur, Freud a posé les premiers jalons pour les investigations suivantes dans le champ des psychoses. La vérification qu“il y a perte d'objet et son incorporation (identification) à l'intérieur du moi par le patient mélancolique avec des conflits d'ambivalence en relation au Surmoi, a permis la dernière étude de Abraham, selon laquelle les mélancoliques retournent à la première phase sadique-anale (per-

Trabalho apresentado ao Congresso Internacional de Psiquiatria, realizado em Paris de 18 a 27 de setembro de 1950.

* Livre docente de Clínica Psiquiátrica da Fac. Med. da Univ. de São Paulo. 
te, élimination de l'objet comme de fèces) et, ensuite, à la deuxième orale (sadique-orale) avec les premières irruptions d'agressivité et d'ambivalence devant l'objet partiel (poitrine) et le totale (mère).

La littérature se rapportant à la psychanalyse des psychoses est déjà très riche, la majorité des auteurs se basant sur les premières recherches de Freud et Abraham. D'un côté, des psychanalystes soutiennent que des modifications techniques ne sont pas nécessaires pour ce qui a trait à l'analyse des neurotiques et d'un autre côté, certains, cependant, prétendent à de profondes modifications. Lorsque nous commençons un traitement psychanalitique à un patient délirant, désagrégé ou intensement inhibé, nous sentons que notre tâche est plus difficile que lorsque nous analysons des patients hystériques, phobiques et obsédés. Le patient refoule partiellement ou totalement la réalité ou, mieux encore, il vit dans une réalité bien diverse de celle où le psychanalyste vit et travaille. La réalité du psychotique s'approche beaucoup, presque à s'y identifier, de la réalité de l'enfant dans ses premières étapes de développement. Il y a perte d'objets du monde extérieur par soustraction et par retraite de la libido objetale qui tend à se fixer dans les objets du monde intérieur, avec des phénomènes de régression de la libido et du moi aux premières phases. Le psychotique cherche à se défendre de ce monde intérieur plein de mauvais objets et est sujet à l'anxiété en face des situations dangereuses les plus variées.

Dans la règle le contact emotionel avec le patient est difficile, comme si le moi n'était pas présent ou alors des réactions d'anxiété et d'agressivité apparaissent à chaque moment empêchant la coopération. Il n'y aurait aucun sens, dans une telle situation, de penser en systématiser la technique. Seule une chose a de l'interêt immédiat: c'est d'entrer en contact affectif avec le malade, sans lequel aucun traitement ne sera possible. Dans notre petite expérience (environ dix cas traités avec un succès relatif et de nombreux autres interrompus) nous eûmes l'occasion d'envisager maintes difficultés dans l'une ou l'autre phase du traitement. Auparavant nous avions déjà médité sur certains aspects de la thérapeutique du choc (la convulsothérapie et les comas insuliniques). Après l'expérience de régression profonde et violente touchant presque au seuil de la mort, les patients, en leur retour à l'état de conscience, tendaient toujours à investir libidinalement les personnes de l'ambiance immédiate, particulièrement le médecin et les infirmiers. L'amélioration du cadre psychopathologique accompagnait de près la prise de conscience et le progressif affermissement du transfert positif, ce qui signifiait l'acceptation d'une bonne réalité externe dans la personne du médecin.

Nous avons orienté notre technique auprès de patients désagrégés et aliénés à l'ambiance dans le but qu'ils prennent pleine conscience de la personne de l'analyste comme bon objet, c'est-à-dire, comme de quelqu'un qui veut l'aider, une telle situation étant posée de façon systématique au patient. Nous sentions toujours que la personne de l'analyste était la principale porte d'entrée, la plus adaptée pour que le patient puisse pénétrer dans le monde extérieur, dans la réalité refoulée. Quel que soit le matériel psy- 
chopathologique présenté nous nous sommes efforcés de le rapporter à la situation de transfert et, quand cela n'était pas possible, nous l'avons laissé de côté, cherchant à insister sur la personne du psychanalyste comme bon objet jusqu'à ce que le patient soit capable d'un contact même très petit avec la réalité. L'anxiété du psychotique en face des mauvais objets de son intérieur et devant la possibilité de perdre les bons ("position dépressive" de M. Klein) s'attenue à mesure que la bonne "imago" de l'analyste est en train de s'interioriser, neutralisant l'action des persécuteurs internes. Toutes les autres interprétations ne réussissent pas dans cette émergence parce qu'elles ne pénètrent pas, seule le transfert actuel par son pouvoir dynanique et affectif étant capable de vaincre les résistances. Il est nécessaire que la patient ressente qu'il existe de bons objets dans la réalité externe, car uniquement l'identification avec eux permettra le travail d'expulsion des mauvais objets de son intérieur et l'atténuation de l'anxiété par la possible perte des hons. Il sentira qu'il les retrouvera dans la personne du médecin, au commencement. L'analyste doit être vigilant en rapport au contre-transfert, principalement le négatif. Attitudes de peur, de précipitation, de long silence, d'impatience et d'autres attitudes négatives de la part du psychanalyste tendent à augmenter l'anxiété et les résistances du patient car il interprétera la situation comme la réponse d'un monde réflexivement agresseur.

Avec cette position technique, l'analyse des psychotiques ne se différenciera pas sensiblement de celle des neurotiques. La finalité, c'est l'acceptation d'une bonne réalité pleine de bons objets, c'est la réconciliation avec la vie. L'orientation technique marche ici très près de nos acquisitions théoriques. Nous savons qu'au début, l'analyse des neurotiques adultes a fait beaucoup de lumière sur les conflits de l'enfant neurotique, et, selon $M$. Klein, l'analyse directe de l'enfant, tend à dépister de plus en plus les cadres psychotiques durant l'enfance. Nous savons aussi que la meilleure et la plus profonde connaissance des conflits entre les divers "noyaux du moi" (Glover) ou entre les premières phases d'intégration du moi avec la vie instinctive (sexuelle et agressive), augmentera nos connaissances sur le dynamisme psychique des psychoses. De toute façon, cela n'influencera pas la technique en rapport spécialement à l'analyse des psychotiques. Introjection et projection, négation, scission (split) de l'objet et du moi, fantaisies de l'omnipotence, les hallucinations primaires de la phase du "moi de plaisir" (Freud) etc., sont des mécanismes psychotiques prédominants. Le psychanalyste cherchera à interpréter les hallucinations et les délires, les phénomènes kinètiques pathologiques et les autres manifestations morbides par les premières expériences dans lesquelles la réalité externe a été deformée au sens de l'impulsivité infantile et des mécanismes de défense déjà mentionnés. Sous cette forme, la symptomatologie psychotique est compréhensible, comme l'a montré Freud, en fonction de phénomènes de régression et de restitution. Certains rêves et fantaisies nous ont suggéré que dans plusieurs symptômes, les psychotiques vont répetant et vivant des impressions très profondes et distantes liées peut-être à la vie intra-utérine (do mouvements et d'autres activités du corps de la mère) et, avec plus de cer- 
titude, aux premières expériences quand les excitations du monde extérieur ont été reconnues comme dangereuses à sa propre préservation. Maintenant, en réflechissant sur la plus grande régression de la libido et du moi dans les psychotiques, nous comprenons la plus grande difficulté du traitement de ces patients en relation aux neurotiques. Cependant, l'investigation psychanalitique en psychiatrie ouvre à notre science un chanp et des perspectives fascinantes que nous devons cultiver pour le bien de nos patients et aussi comme contribution à la continuation de l'œuvre que nous a légué la personalité géniale de Sigmund Freud.

\section{RESUME}

L'auteur se réfère brièvement aux premières études de Freud et Abraham sur l'investigation psychanalitique des psychoses. Comme le neurotique, le psychotique a de la capacité de transfert. Cependant le contact affectif est difficilement établi avec les patients délirants, désagrégés et intensement inhibés. Dans sa petite expérience (environ dix cas traités avec un succès relatif et de nombreux autres interrompus), l'auteur a rencontré mainte difficultés dans l'une ou l'autre phase du traitement. Étudiant certains aspects psychologiques des patients soumis aux techniques du choc (la convulsothérapie et leś comas insuliniques), l'auteur a ressenti l'extraordinaire importance de la personne dù médecin comme la principale porte d'entrée dans la réalité extérieure. Il a développé la technique de fortifier le transfert positif et de discuter de façon systématique avec le patient, la personalité de l'analyste pour qu'il puisse prendre conscience du monde extérieur et de sa situation. Il fait d'autres considerations sur les mécanismes plus profondes de cette technique et de sa valeur dans la psychanalyse des psychotiques.

\section{RESUMO}

A verificação de que não há diferença fundamental entre neurose e psicose abre largas perspectivas para a aplicação do método psicanalítico em psiquiatria. Como o neurótico, tem o psicótico capacidade de transferência, o que torna possível seu tratamento. Após referir os primeiros estudos de Freud e Abraham sôbre a psicanálise das psicoses, aborda o autor o problema da técnica. Em pacientes delirantes, desagregados ou fortemente inibidos há, de regra, grande dificuldade no estabelecimento do contacto emocional necessário para o tratamento. Em sua pequena experiência (10 casos tratados com relativo êxito e outros tantos interrompidos) encontrou o autor tais dificuldades em uma ou outra fase do tratamento. Após observações feitas em pacientes submetidos às técnicas de choque, em que, ao recuperarem o estado de consciência, tendiam a se derramar libidinalmente sôbre o médico ou enfermeiros, a cura se processando em relação direta com a conscientificação e com o fortalecimento da transferência positiva, orientou o autor sua técnica no mesmo sentido. Em seu trabalho com psicóticos desagregados e inibidos, distantes da realidade externa, procurou manter e for- 
talecer o contacto emocional positivo, a princípio conscientificando ao máximo a personalidade do analista. Todo o material era imediatamente reduzido a tal situação, o psicoterapeuta sendo considerado e manipulado como a principal porta de entrada para o mundo. Sua boa "imago" como representante e símbolo dos bons objetos da realidade externa, tendia a neutralizar progressivamente a ansiedade do psicótico ante os maus objetos interiorizados, os perseguidores internos. As resistências, compreendidas em função dos mecanismos de defesa predominantemente psicóticos, são conscientificadas e trabalhadas simultânea ou anteriormente às tentativas de redução histórica das experiências psicóticas atuais. A mais profunda regressão da libido e do ego dêsses pacientes explica a maior dificuldade do tratamento em relação ao neurótico. Todavia, a investigação psicanalítica em psiquiatria deve ser cultivada a bem dos nossos pacientes e como contribuição para o prosseguimento da obra que nos legou Sigmund Freud.

R. Araujo, 165 - São Paulo, Brasil. 